\title{
Application of the LMS algorithm to identify the surface velocity responsible for the radiated sound pressure
}

\author{
Wolfgang Kropp ${ }^{1, *}$, Krister Larsson ${ }^{1}$, Thiago Lobato ${ }^{2}$, and Roland Sottek ${ }^{2}$ \\ ${ }^{1}$ Applied Acoustics, Chalmers University of Technology, 41296 Göteborg, Sweden \\ ${ }^{2}$ HEAD acoustics GmbH, 52134 Herzogenrath, Germany
}

Received 11 March 2021, Accepted 10 June 2021

\begin{abstract}
A time domain approach based on the least mean square (LMS) algorithm is applied to reconstruct the source amplitude and source distribution on a plate. For this a numerical experiment is established. A boundary element model is used to calculate the required impulse response functions describing the pressure in near- and far-field for a given volume flow at individual patches on the plate. Three different cases are considered. Firstly, a volume flow is given to a single patch. The LMS algorithm is used to reconstruct the source signal by means of receiving positions in the far-field. Secondly, the approach is used to identify the vibration pattern and source signal on a line of patches. Thirdly, a vibration pattern was given to the plate as the whole. For the reconstruction an assumption was made about the underlying vibration patterns (e.g. expansion in vibrational modes). Such an approach proved to be very time efficient and powerful. It also showed the need to place the receiving positions in the near-field to be able to obtain correct results over the whole frequency range. However, this is not a problem of the approach based on the LMS algorithm, but just due to the underlying physics. It is not possible to deduce the near-field from far-field observations, and therefore the vibrations mainly leading to a near-field are simply not visible in the far-field.
\end{abstract}

Keywords: Source identification, Inverse problem, Time domain

\section{Introduction}

For the successful design of sound and vibration properties of products and environments, experimental work will be indispensable even in the foreseeable future. One of the main tools in this context is the so-called transfer path analysis (TPA). Classical TPA dates back to the early eighties when Verheij measured indirectly the forces at the interface between vibration isolators, engine and ship hull (see e.g. [1]). Since the early eighties different approaches have been made to develop the TPA towards a tool applicable in industry. A multitude of different methods have emerged whose similarities, but also differences can easily be perceived as confusing. In [2] an attempt is made to classify the existing methods as "classical TPA", "component-based TPA" and "transmissibility-based TPA" where the latter also includes the operational transfer path analysis. The authors in [2] conclude that the three families are highly interrelated and that little has been suggested about the methods' relative success for typical industrial cases and sensitivity to various sources of error. Further research, in both numerical simulation and experimental validation, would substantiate this TPA framework in that respect.

*Corresponding author: Wolfgang. Kropp@chalmers.se
In the special case of airborne sound source characterisation (i.e. reconstructing the velocity distribution on radiating surfaces) different methods have been developed over the recent years. Methods such as the near-field acoustic holograpy (NAH) [3, 4], the inverse boundary element method (IBEM) (e.g. [5] and the least squared methods (LSM) (see e.g. [6, 7]) are used in this context. The NAH demands special geometries to allow the application of the Fourier transform. The IBEM is more general in geometry but is sensitive to similar errors as the TPA since an inversion has to be carried out.

Typical sources of errors are:

- Insufficient signal to noise ratio which might lead to erroneous observation of field quantities (e.g. measured vibrations or sound pressures) or frequency response functions (FRFs). Measurement noise in some elements of e.g. a transfer matrix is distributed over all elements after inverting the matrix.

- Non-accurate FRFs due to e.g. small misplacement of transducers or the inherent difficulties to measure certain degrees of freedom (DoF) such as rotational degrees.

- Not correct or not complete description of the interfaces as part of the transfer paths between components. Neglecting transfer paths can result in an erroneous result for the transfer paths considered. 
- Neglecting the presence of correlated multiple sources. Multiple correlated sources might lead to "crosstalking" at interfaces. Neglecting some of the sources will give erroneous results for those sources taken into account.

- Performing the operational test in an environment different from the targeted assembly. This only leads to correct results when the mounting condition does not influence the source itself. E.g. the blocked-force [8] and free-velocity [9] methods provide two extreme boundary conditions for the description of an active component. The boundary condition in the case of the hybrid interface method [10] depends on the dynamic stiffness of the test rig. The different boundary conditions will influence the active component differently.

- And finally just the limitation by lack of information due to the physics of the problem might not allow for identifying sources or transferpaths from measurements in the far-field accurately.

The scope of this paper is to demonstrate the capability of the LMS algorithm to identify the surface velocity responsible for the radiated sound pressure and to investigate the influence of different parameters such as background noise or accuracy of transfer function as well as limitations by physics on the quality of the results. In practical applications typically measurements of sound pressure signals in a multitude of receiving positions are carried out. At the same time a set of transfer functions from the anticipated source positions to the receiver positions is measured. In this way an inverse problem is established which has to be solved. Among the solving methods discussed in literature the overwhelming majority is formulated in the frequency domain and involve matrix inversion of different types. The inverse problem is well known to be ill-posed and has been shown to be sensitive to measurement noise and to suffer from numerical ill-conditioning at frequencies associated with the natural frequencies of the components [11]. Usually, the robustness of such solutions has to be improved by applying some form of regularization [12]. One usual attempt to cure problems is to create an overdetermined equation system and use truncated singular value decomposition [13].

In the following a different approach is utilised based on work originally presented in [14]. There two of the authors presented an approach based on the LMS algorithm in the time domain. They demonstrated the approach which is explained in more detail in Section 1.2 for a single input multiple output system (SIMO) where a force is exciting a structure in a known excitation position. The approach showed to be very robust when reconstructing forces acting on a beam. Although not included in the paper [14] but presented at the conference they also extended the approach to a multiple input multiple output system (MIMO system), i.e. excitation with several forces in known excitation positions. This extension was later published in [15] and applied in [16] for the indirect measurement of walking forces on wooden floors.

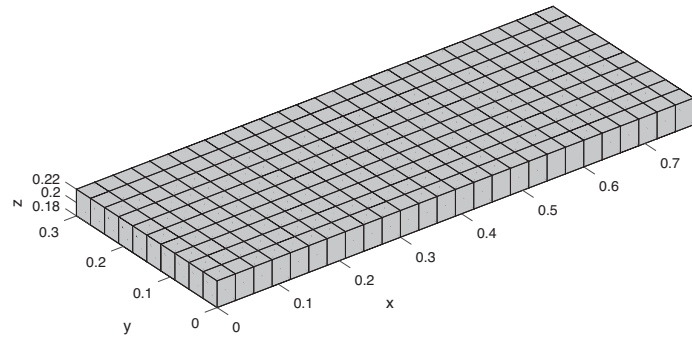

Figure 1. Geometry of the plate used in the numerical experiments.

\subsection{The setup}

To investigate the possibilities to reconstruct the velocity distribution on a surface a numerical experiment is set up consisting of a $4 \mathrm{~cm}$ thick plate with the length of $0.78 \mathrm{~m}$ and the width of $0.3 \mathrm{~m}$ as shown in Figure 1.

The plate is situated at a height of $30 \mathrm{~cm}$ over an infinite and rigid ground. The plate surfaces are divided into quadratic shaped elements of the size of $3 \mathrm{~cm}$ times $3 \mathrm{~cm}$. At the nodes of each element a velocity in normal direction of the surface is prescribed which determines the volume flow created by each element.

To calculate the radiation from the source distribution on the plate a half space BEM formulation is used based on the work by Brick presented in [17]. The sound pressure is evaluated at 321 receiving positions on a half sphere with a radius of $1 \mathrm{~m}$ around the origin and 260 positions situated directly $6 \mathrm{~cm}$ in front of each of the elements on the top surface of the plate (see Fig. 2).

From the radiated pressure transfer functions between each element on the top surface and all receiving positions are calculated by normalising the calculated pressure values by the volume flow at the surface. Typical results for the transfer functions are shown in Figure 3.

The transfer functions to the positions close to the plate are relatively smooth over frequency and differ mainly in level. For positions on the sphere interference effects with strong dips in the FRF can be observed. From these FRFs the impulse response functions (IRFs) are calculated by inverse Fourier transform. In the following a maximum frequency range of $1500 \mathrm{~Hz}$ (i.e. a sampling rate of $3000 \mathrm{~Hz}$ ) is considered. With 200 frequency lines the frequency resolution is $7.5 \mathrm{~Hz}$.

\subsection{The LMS algorithm applied to source identification}

Today the LMS algorithm is a standard tool in filter design. It has been invented by Widrow and Hoff [18] and was the basis for their work with neural networks and machine learning. By means of the LMS algorithm a desired filter is approximated by determining a limited number of coefficients of a new filter that results in the least mean square of the error signal, i.e. the difference between a desired and the actual signal observed. At each time step the LMS algorithm follows the steepest gradient in order to minimise this error. The result converges to the optimal 


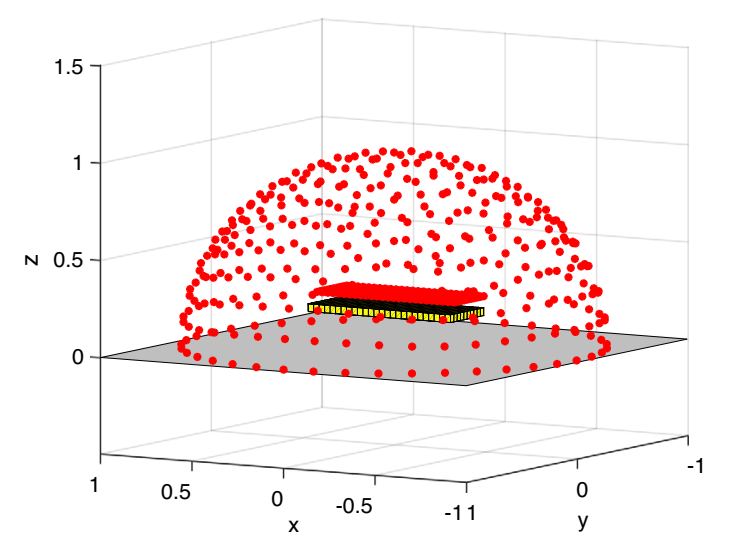

Figure 2. The plate above the ground in combination and two sets of receiving positions. One set directly above the plate and one set distributed on a sphere.

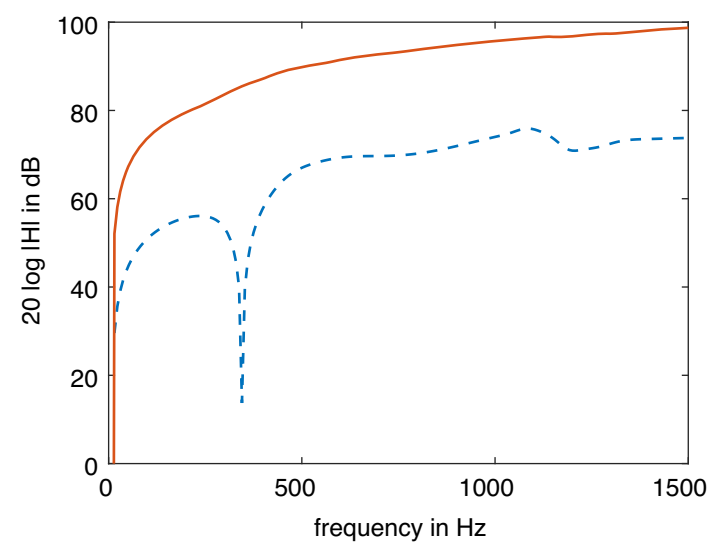

Figure 3. Example for transfer functions for the radiation of one patch on the plate to a position directly above the plate (solid line) and on the half sphere (dashed line).

filter, the so-called Wiener-Hopf filter. Figure 4 shows a typical situation where the output $a_{d}(n)$ of a system described by the IRF $h_{o}$ should be reconstructed by a filter $h$ with $I$ coefficients.

By comparing the output $a_{d}(n)$ of the system with the output from the filter $\mathbf{h}$ the error $e(n)$ at each time sample $n$ can be calculated as,

$$
e(n)=a_{d}(n)-\mathbf{h x}(n),
$$

where $x$ is the observed input into the system $(x=[x(n)$, $\left.x(n-1), \ldots, x(n-I+1)]^{T}\right)$ with $T$ indicating the transposed form of $x$ and $\mathbf{h}$ the unknown filter $(\mathbf{h}=[h(0), h(1)$, $\ldots, h(I-1)])$.

The expectation value of the squared error is then,

$$
E\left[e(n)^{2}\right]=E\left[\left(a_{d}(n)-\mathbf{h x}(n)\right)^{2}\right] .
$$

The main idea of the LMS algorithm is to minimise the mean value of the quadratic error. As this is a quadratic equation the minimum is found by following the gradient of Equation (2) with respect to the filter coefficients $h_{i}$,

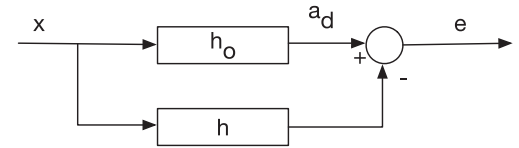

Figure 4. Typical task for filter design with $x$ as input signal, $h_{o}$ the desired impulse response function of the filter, $h$ the designed filter and $e$ the error between desired signal $a_{d}$ and the signal created by the filter $h$.

$$
\frac{\partial E\left[e(n)^{2}\right]}{\partial h_{i}}=2 E\left[e(n) \frac{\partial e(n)}{\partial h_{i}}\right]=-2 E[e(n) x(n-i)] .
$$

Starting from this Widrow and Hoff showed that instead of calculating the expectation value at each time step the gradient can be calculated as $e(\mathrm{i}) x(n-i)$ which leads to an adaptive process where,

$$
\mathbf{h}(n+1)=\mathbf{h}(n)+2 \alpha e(n) \mathbf{x}^{T}(n),
$$

and that this iterative process converges towards the results obtained by (3). $\alpha$ is the step size following the gradient in the adaptive process for calculating the filter coefficients. To ensure stability Widrow suggested in [19] as a "rule of thumb" that the step size has to obey the condition,

$$
\alpha<\frac{1}{I E\left[x(n)^{2}\right]},
$$

where $E\left[x(n)^{2}\right]$ is the mean square value of the reference signal.

The frequent use of the LMS algorithm for filter design in e.g. active noise control is based on the fact,

- That it does not demand a matrix inversion and, therefore the solution becomes more robust in the case of low SNR and ill-conditioned systems.

- That the gradient is determined by the absolute error $e(n)$. This means that the error due to strong components in the signals (e.g. due to resonances) governs the gradient rather than weak components (e.g. anti resonances). This circumstance makes the algorithm very robust with respect to background noise.

- And that the algorithm is extremely simple to implement.

These properties make the LMS algorithm also an interesting approach for the reconstruction of input signals into systems described by impulse response functions. As the convolution is a commutative operation the only minor modification is the exchange of $\mathbf{x}(n)$ and $\mathbf{h}$, i.e. make $\mathbf{h}$ to the input and $\mathbf{x}(n)$ to the function to be updated as shown in Figure 5.

The equation corresponding to Equation (4) is therefore,

$$
\mathbf{x}(n+1)=\mathbf{x}(n)+2 \alpha e(n) \mathbf{h}^{T}(n) .
$$

The main difference is that $\mathbf{h}(n)$ is of finite length and always used completely. This has two consequences. 


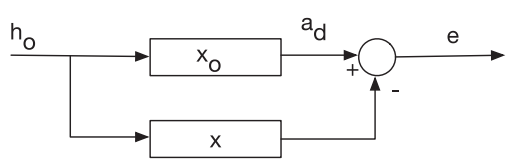

Figure 5. Adaptive process to determine the unknown excitation $x(n)$.

Firstly, the reconstructed signal $\mathbf{x}(n)$ is erroneous as long as $n$ is smaller than the length of $\mathbf{h}$. Consequently, the length of the desired/observed signal $a_{d}(n)$ has to be at least twice the number of coefficients of $\mathbf{h}$ to capture the response to an excitation completely at least once.

Secondly, the time iterative process described by Equation (6) might be too short to converge to a sufficient accurate result. Therefore the process is repeated several times using the result of $\mathbf{x}$ of the previous run as start values.

The formulation in (4) is for a single input single output system (SISO). It easily can be extended for a single input multiple output (SIMO) system which would be the case in an overdetermined system. For a system with $R$ output signals, Equation (1) yields the error at each receiving position as,

$$
e_{r}(n)=a_{d, r}(n)-\mathbf{h}_{r} x(n) .
$$

$a_{d, r}$ is the measured signal at each receiving position $r$ and $\mathbf{h}_{\mathbf{r}}$ the IRF from the excitation position to the receiving positions $r$. The gradient can be determined in analogy to (4) and the iterative updating procedure yields,

$$
\mathbf{x}(n+1)=\mathbf{x}(n)+2 \frac{1}{R} \sum_{r=1}^{R} \alpha_{r} e_{r}(n) \mathbf{h}_{\mathbf{r}}(n) .
$$

By this the mean gradient is calculated. From the equation it is obvious that always the strongest absolute error will determine the gradient most. Extending the problem from a SIMO system to a MIMO system (i.e. multiple input and multiple output system) is a further but straight forward step. The error in this case is calculated as before as the deviation between the measured signal $a_{d, r}$ and the predicted signal. However, as several sources are acting the predicted system at each receiving position is a superposition of the responses due to all sources, i.e.,

$$
e_{r}(n)=a_{d, r}(n)-\sum_{i=1}^{I} \mathbf{h}_{i, r} \mathbf{x}_{i}(n) .
$$

The iterative updating is done for each source separately,

$$
\mathbf{x}_{i}(n+1)=\mathbf{x}_{i}(n)+2 \frac{1}{R} \sum_{r=1}^{R} \alpha_{r} e_{r}(n) \mathbf{h}_{r, i}(n)
$$

The implementation of Equations (6)-(10) is rather simple resulting in just a few lines of code which also is a strength of the LMS algorithm.

\section{Numerical experiments}

Numerical experiments allow under controlled conditions to investigate the potential to reconstruct a given

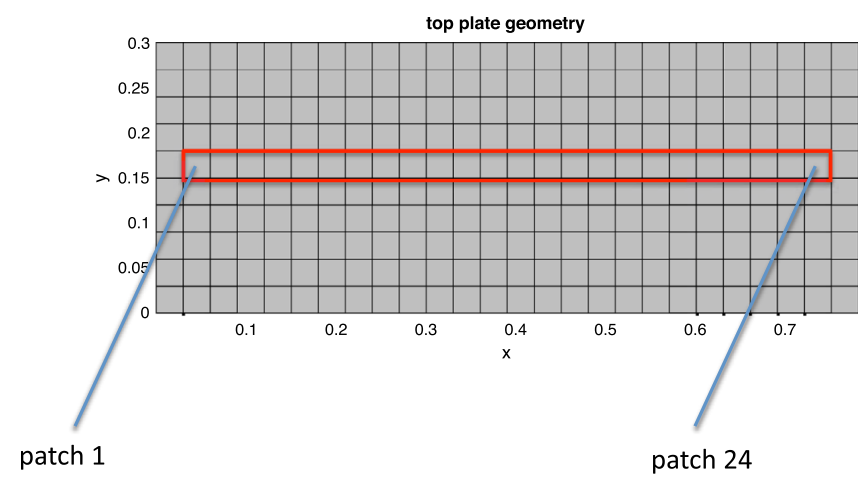

Figure 6. Position of possible sources in front of the plate.

source distribution on an object by means of the approach based on the LMS algorithm as described in the previous section. Based on the numerical experiments for the plate presented in Section 1.1 a systematic study is presented in the following starting from a single point source and extended to more complex source distributions. To limit the complexity for the beginning only patches along a line as indicated in Figure 6 are considered. The patches are numbered from 1 to 24 . When prescribing a velocity to a patch it is defined in the four nodes around the patch. The velocity in the normal direction to the surface in the centre of the patch is calculated as average of the velocity at the four nodes. As a consequence even neighbour patches will have a velocity as they share nodes with the patch where the velocity is prescribed.

\subsection{A single source}

In order to demonstrate the functioning of the LMS algorithm the most simple case of only one source (at patch 14) and one receiver is studied first (a SISO system). The source signal consists of white noise, but any other signal could have been chosen. As receiving position one of the positions on the half sphere is selected. It is assumed that the source position is known. For this case a reconstruction of the source signal is carried out. The results of the original time signal of the source and the reconstructed time signal after 800 repetitions of the updating process are shown in Figure 7 . The iteration process could be interrupted when the error $e$ becomes smaller than a certain limit or when the change in the error between two subsequent iteration steps is smaller than a given value. In this paper a limit of 800 iteration steps was used for all cases.

As the length of the IRFs is 400 samples the reconstructed values for the sources signal for the first 400 samples are erroneous. After 400 samples the agreement between original and reconstructed signal seems to be fine, although such judgements are very difficult to make by means of inspecting the time records. Instead the autospectra of both signals (only for valid samples, i.e. higher than 400) are shown in Figure 8.

From the figure the good agreement is visible over a wide frequency range which is not too surprising as the data used in the simulation are free from noise and the source 


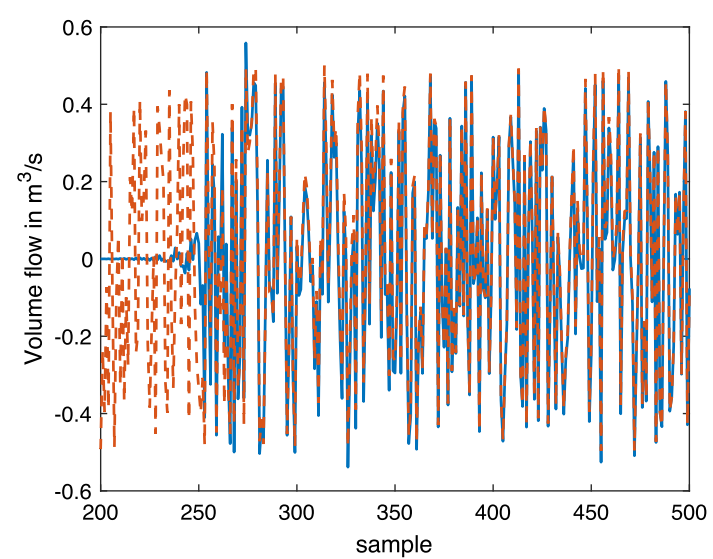

Figure 7. Original source signal (dashed line) and reconstructed source signal (solid line).
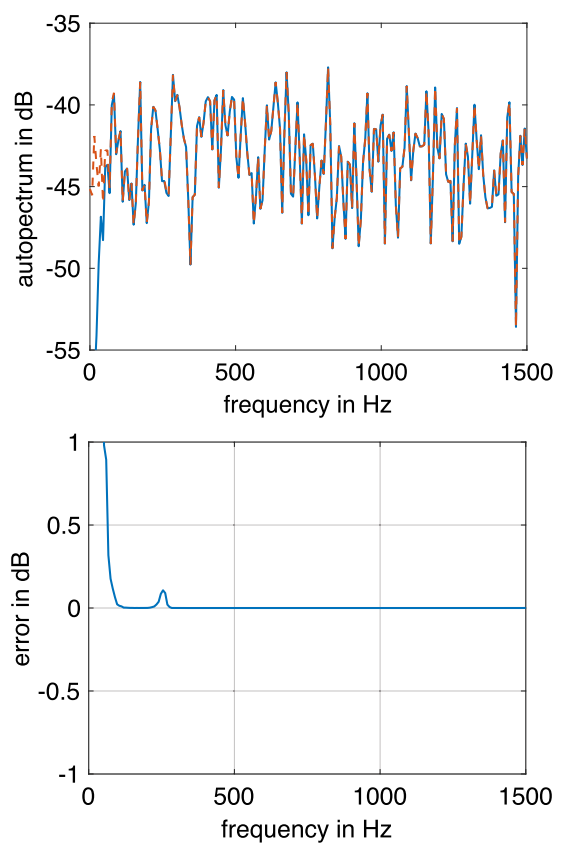

Figure 8. The upper curve shows the comparison of the original source spectrum (dashed line) and reconstructed source spectrum (solid line). The lower curve shows the difference between original source spectrum and reconstructed source spectrum.

position is known. However, at very low frequencies there is a substantial deviation. The reason is the low response in the FRF (see Fig. 3). As the gradient is determined by the absolute error small values in the FRF will lead to small values in the pressure signals. As weak pressure signals hardly influence the adaptive process such frequency ranges are not updated properly. This can be cured as usual when applying the LMS algorithm by only focusing on the frequency range where a good adaptation is needed. Figure 9 shows the resulting error when applying a low pass filter with a cutoff frequency of $200 \mathrm{~Hz}$ to the input signal, i.e. the IRFs.

Now the signal below $200 \mathrm{~Hz}$ controls the adaptive process. Consequently the results are improved at low

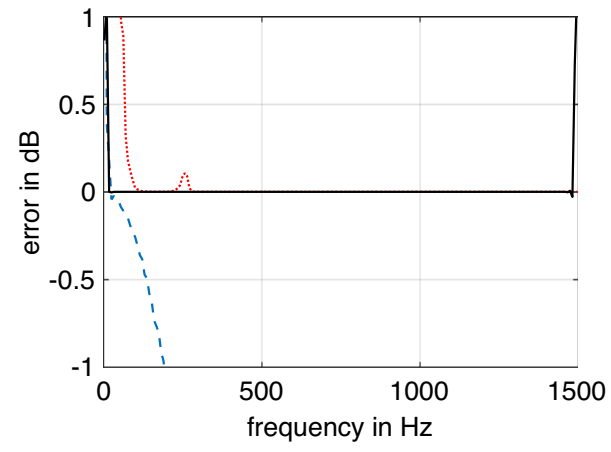

Figure 9. Error in the reconstructed source spectrum for the original IRFs (dotted line), after applying a lowpass filter to the IRFs with a cut off frequency of $200 \mathrm{~Hz}$ (dashed line) and after integrating the IRFs (solid line).

frequencies but are erroneous above $200 \mathrm{~Hz}$. The low response of the FRFs at low frequencies is based on the underlying physics as the sound pressure radiated from volume sources is proportional to the volume acceleration, i.e. the time derivative of the volume flow $\dot{Q}$. Carrying out a time integration of the IRFs compresses the dynamics and the FRFs become more flat. Applying the integrated IRFs leads to that the reconstruction will be successful over more or less the whole frequency range as shown in Figure 9. Derivation with respect to time applied to the reconstructed source signals will correct such a choice of IRFs.

In the following the integrated IRFs are used throughout the paper.

\subsubsection{The influence of measurement noise}

A usual problem when using the TPA approach is that the observations (i.e. the measured pressure signals) are disturbed by measurement noise. Therefore, the effect of measurement noise on the performance of the LMS algorithm is investigated in the following.

In typical TPA approaches the influence of measurement noise is reduced by formulating an overdetermined system (SIMO), i.e. more receiving positions than sources are used for the reconstruction of the source properties. In the case here the pressure signals in several receiving positions on the half sphere are used. To demonstrate the functioning of the LMS algorithm in connection with an overdetermined system, noise is added to the simulated pressure signals at the receiving positions. The amplitude of the noise sequences is chosen so that for each receiving position a prescribed signal to noise ratio (SNR) is obtained. The number of receiving positions $(N r)$ in the half sphere is chosen as $N r=2^{n}$ with $n=0,1, \ldots, 8$. Figure 10 shows the results from this study in the form of the error calculated from the ratio between the energy in the reconstructed source signal $Q_{r}$ and the energy in the original source signal $Q_{o}$,

$$
\Delta L=10 \log \frac{Q_{r, \mathrm{rms}}^{2}}{Q_{o, \mathrm{rms}}^{2}}
$$


The error will depend on the SNR, the number Nr of receiving positions, but also on the selection of the receiving positions. Therefore it is necessary to calculate the error for a number of realisations with different receiving positions. 64 realisations are used for each combination of SNR and $\mathrm{Nr}$ and the results for each combination are represented as boxplot in Figure 10. The median is indicated by a notch and the edges of the box represent the 25th and 75th percentiles respectively. The whiskers are extended to the most extreme data points the algorithm considers to be not outliers, and the outliers (light grey) are plotted individually.

From the figure it is visible that the increase of receiving positions in the reconstruction process is able to suppress the negative impact of measurement noise. For a SIMO system the gradient in the adaptive process is calculated as the average over the error in all receiving positions. As the added noise is uncorrelated, averaging will suppress the influence of measurement noise on the gradient. In addition, in the time iterative procedure errors in the gradient will lead that the algorithm will march into a somewhat erroneous direction. However, in the next time step the error in the gradient will be different and therefore also the error in the direction. Therefore as long as the error is random, uncorrelated to the input signal and not too big the adaptive process will in any case converge on a random path towards the correct value. However, it will never reach the correct value exactly. How close the value will come to the true value is depending on the SNR and the degree of overdetermination.

The results show that already for $N r=16$ receiving positions the error $\Delta L$ can be kept below $0.5 \mathrm{~dB}$ in the case of an $\mathrm{SNR}=0 \mathrm{~dB}$. In Figure 10 analytical results are plotted describing the influence of averaging on the error $\Delta L$ as function of the SNR,

$$
\Delta L=10 \log \left(1+10^{\frac{-0.1 \mathrm{SNR}}{N r}}\right) .
$$

There is a good agreement between the simulation results and Equation (12), indicating that the use of the LMS algorithm in connection with an overdetermined system is actually equivalent to averaging out the noise contribution and that the number of receiving positions is equivalent to the number of ensembles averaged over in Equation (12).

These results concern the overall energy in the spectrum. In the case of individual frequency components, however, there can be considerable deviations. To evaluate the similarity between the original and reconstructed source spectrum, an approach similar to the Modal Assurance Criterion (MAC) [20] is applied. The MAC value is widely used in modal analysis and is a metric that calculates the degree of linear similarity between two mode shapes. Using the same mathematical definition of the MAC we define here the Spectral Cross Correlation (SCC), which describes the similarity between two complex spectra. This enables a very robust similarity estimate including the spectrum shape and its energy content. The SCC value varies between 0 and 1 and it is defined as the

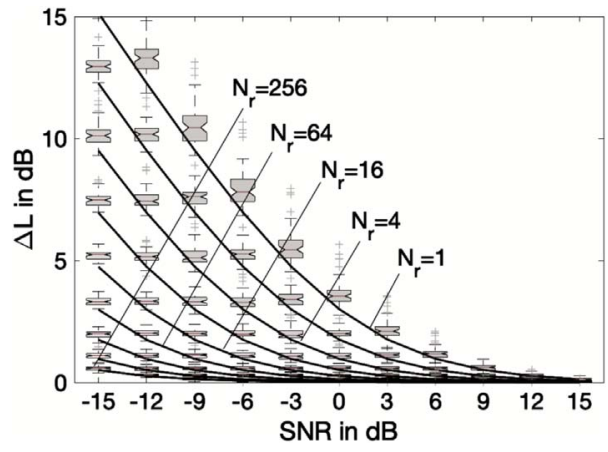

Figure 10. Error according to Equation (11) in the prediction as function of receiving positions and signal to noise ratio shown as boxplots. The results are compared with the error predicted by Equation (12) (solid lines). The upper curve corresponds to 1 receiving position. The consequent curves correspond to $2,4,8$, $16,32,64,128$ and 256 (lowest curve) receiving points.

normalized cross-correlation of the two spectra. It is defined in Equation (13) (where $(\cdot)^{H}$ is the Hermitian-transpose operator),

$$
\operatorname{SCC}\left(\mathbf{S}_{\mathbf{o}}, \mathbf{S}_{\mathbf{r}}\right)=\frac{\left|\mathbf{S}_{\mathbf{o}}^{H} \mathbf{S}_{\mathbf{r}}\right|^{2}}{\left(\mathbf{S}_{\mathbf{o}}^{H} \mathbf{S}_{\mathbf{o}}\right) \cdot\left(\mathbf{S}_{\mathbf{r}}^{H} \mathbf{S}_{\mathbf{r}}\right)}
$$

In Figure 11 a similar tendency for the MAC can be seen, in which the more similar the spectra are in shape, the higher the number of receiver positions and the SNR.

\subsubsection{Wrongly anticipated source location}

The application of the LMS algorithm demands a correct assumption of the position of the source in order to establish the appropriate IRFs. In typical real case applications the source positions might often not be known exactly. The consequence might be an erroneous reconstruction of the source strengths. This is investigated in the following. Therefore, it is assumed that patch 14 (see Fig. 6) is the source while in reality the source is placed on different positions. The position was varied between patch 13 to patch 9 , which corresponds to a misplacement by $3 \mathrm{~cm}$ to $15 \mathrm{~cm}$. As expected with increasing distance the reconstruction of the source strength became increasingly erroneous over a wide range of the frequency spectrum. However, below about $500 \mathrm{~Hz}$ the error stayed rather small. Only one receiving position was chosen. To investigate the influence of more receiving positions the biggest distance (i.e. $15 \mathrm{~cm}$ misalignment) was chosen and the number of receiving positions for the reconstruction was gradually increased (Fig. 12). As the number of considered receiving positions increased the error decreases clearly.

However, even for the case of evaluating two receiving positions only, similar good results have been obtained as for eight positions in Figure 12 for frequencies below $800 \mathrm{~Hz}$, depending on the selected receiving positions utilised for the reconstruction. Closer investigations showed that it is more important to select proper positions rather than many. 


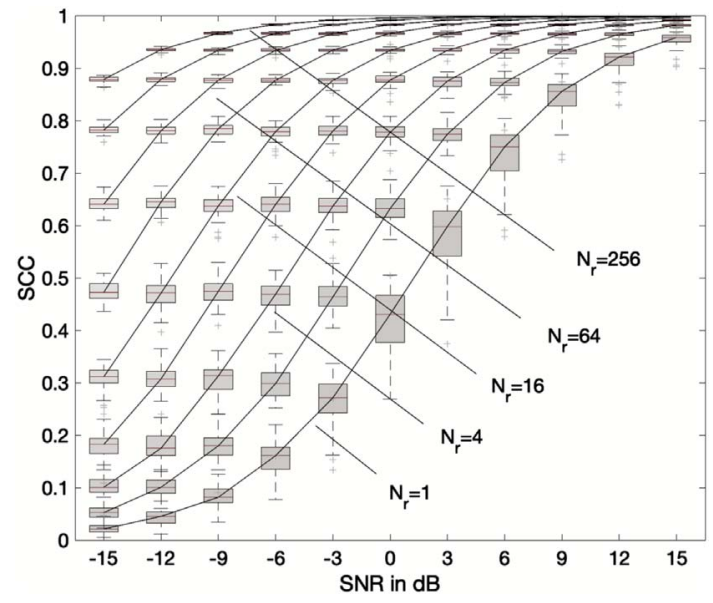

Figure 11. SCC between the original spectrum and the reconstructed spectrum of the source patch according to Equation (13) in the prediction as function of receiving positions and signal to noise ratio shown as boxplots. The curve indicates the median values. The lowest curve corresponds to 1 receiving position. The consequent curves correspond to $2,4,8,16,32,64$, 128 and 256 (highest curve) receiving points.

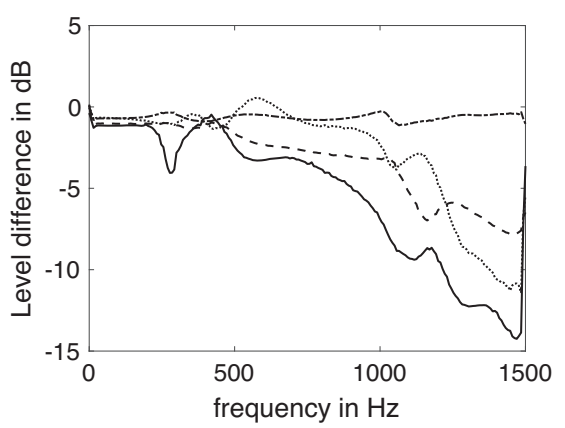

Figure 12. Level difference between prescribed pressure signals and pressure signal which is obtained for the reconstructed source signal assumed at erroneous position. The calculations are carried out for $1(-), 2(\ldots), 4(-)$, and $8(-.-)$ receiving positions.

However, having many positions increases the chance that among these there are sufficient many relevant positions for reconstructing the source strength correctly, even if the source position is assumed $15 \mathrm{~cm}$ apart from the correct position which corresponds to a positioning error of more than $60 \%$ of the wavelength at the highest frequency considered here. Figure 13 shows that the spread is rather small for different randomly selected sets of receiving positions as long as there are sufficient (in this case eight) positions.

From the results we can conclude that even with assuming wrong source positions it is possible to reconstruct the source signal rather accurately. However, these are also bad news as it might implicate that it could be difficult to reconstruct the spatial source distributions correctly as the space information does not seem to play a sufficiently important role at lower frequencies. This will be investigated in the next section.

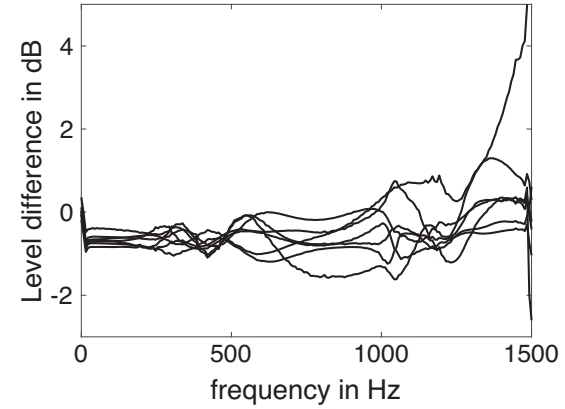

Figure 13. Level difference between prescribed pressure signals and the pressure signal which is obtained for the reconstructed source signal assumed at an erroneous position. The results are presented for seven simulation runs with different eight randomly selected receiving positions.

\subsection{Identification of vibration patterns}

Up to now only a SISO or a SIMO system has been investigated. In the following this is extended to a MIMO system by including all patches indicated in Figure 6 (i.e. marked with a red line) as sources. Two cases are investigated. In the first case the patches are treated as individual sources as part of a line array. For each individual source the strength is identified by the LMS algorithm.

In the second case the individual sources are collected to global vibration patterns along the line array. The source strength of each of these patterns is then determined by the LMS algorithm. This method is shown to be faster. However, it demands some pre-knowledge about the source distribution to chose these patterns in an appropriate way.

\subsubsection{A line array of correlated pistons}

The prescribed source signals at each patch are correlated but different in amplitude. As time signal a white noise sequence is used. The velocity $v_{m}$ at each patch $m$ is described as,

$$
v_{m}\left(N_{p}\right)=A \sin \left(\frac{N_{p} \pi m}{23}\right),
$$

where $A$ is the amplitude of the pattern and $N_{p}$ the number of half wavelength fitting to the whole length of the strip. $N_{p}$ is varied between 1 and 5 . In this way (14) might represent the modal pattern for e.g. a simply supported beam.

For the reconstruction of the velocity fields 40 receiving positions on the half sphere are utilised. Figures 14 and 15 show typical results for $N_{p}=3$ and $N_{p}=5$. The simulations show good agreement above a certain frequency. Below this frequency the results are erroneous. With increasing $N_{p}$ this frequency is increasing as shown in Figure 16. There the spatial averaged squared velocity for the prescribed velocity pattern and the reconstructed pattern are compared. The results are expressed as relative error in decibel for $N_{p}=1$ to $N_{p}=5$. In the figure also the so-called critical frequencies $f_{c}$ in accordance to Table 1 are indicated as 

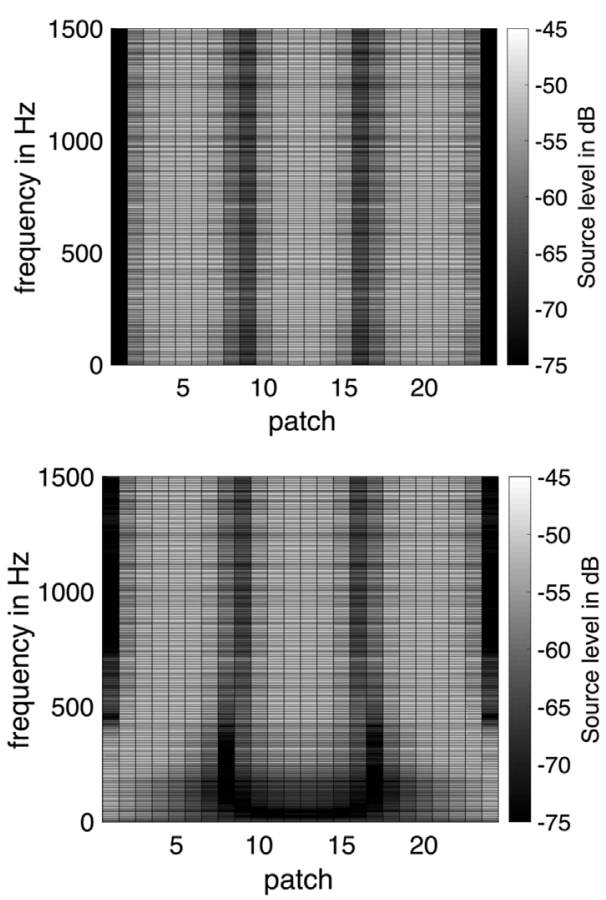

Figure 14. Comparison of the original source distribution along the 24 patches (upper) and the reconstructed source distribution (lower) as function of frequency for $N_{p}=3$. Reconstruction based on far-field receiving positions.

small circles. The critical frequency $f_{c}$ as used here is defined as the frequency where the wavelength in the surrounding fluid (i.e. air) is identical with the prescribed wavelength on the plate.

Inspecting Figures 14 and 15 one can argue that below $f_{c}$ only the part of the prescribed velocity field can be observed in the far-field which is responsible for the radiated sound field. The part of the vibration resulting in a near-field mainly cannot be observed in the far-field positions. The reconstructed source distribution show strong contributions at both ends of the strips where the actual velocity is close to zero. However, we know from theory that for instance for a radiator in a rigid baffle the radiation takes place at the edges/ends of the radiator (see e.g. [21]) at frequencies far below the critical frequency. In this way the results might correctly describe a possible source distribution explaining the radiated field although is not identical with the prescribed velocity. This is further discussed in Section 3.

If these considerations are correct it should be possible to identify the prescribed velocity exactly by using receiving positions in the near-field directly above the plate as shown in Figure 2 and indeed the so reconstructed field is more or less identical with the prescribed velocity distribution (see Figs. 17 and 18).

The only bigger deviations are in the nodal lines where the velocity values are very small and therefore the LMS approach might have difficulties to converge to the correct values. However, this is hardly visible in the figures and does not play an important role for the sound radiation
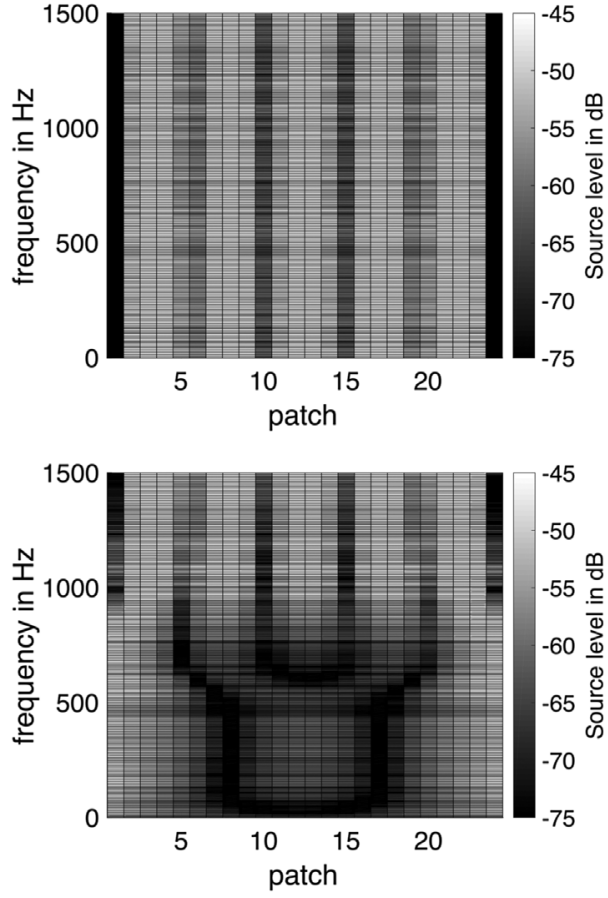

Figure 15. Comparison of the original source distribution along the 24 patches (upper) and the reconstructed source distribution (lower) as function of frequency for $N_{p}=5$. Reconstruction based on far-field receiving positions.

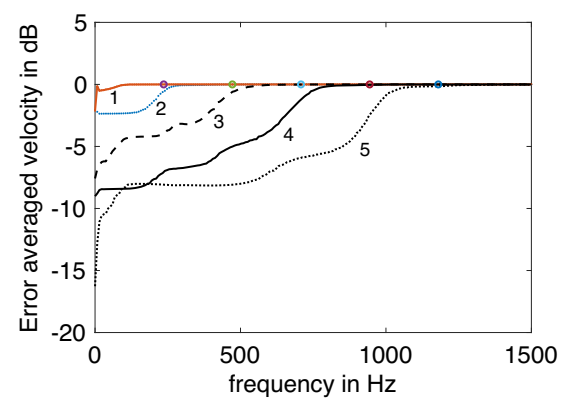

Figure 16. Error in the averaged velocity between original and reconstructed source distribution. Circles indicate the critical frequencies as described in Table 1 . The numbering of the curves corresponds to $N_{p}$.

as the values are very small. In any case one can conclude that the vibration distribution on the plate can be correctly reconstructed when using near-field receiver positions, but this might not be possible at frequencies below the critical frequencies of the underlying vibration patterns when using far-field positions.

\subsubsection{Identification of predefined source configurations}

The alternative to the identification of the source distribution for individual patches is to assume certain patterns from the very beginning. Often the vibration distribution 
Table 1. Critical frequencies for the first five vibration patterns according to Equation (14).

\begin{tabular}{lccccc}
\hline$N_{p}$ & 1 & 2 & 3 & 4 & 5 \\
\hline$f_{c}(\mathrm{~Hz})$ & 236 & 472 & 708 & 944 & 1180 \\
\hline
\end{tabular}
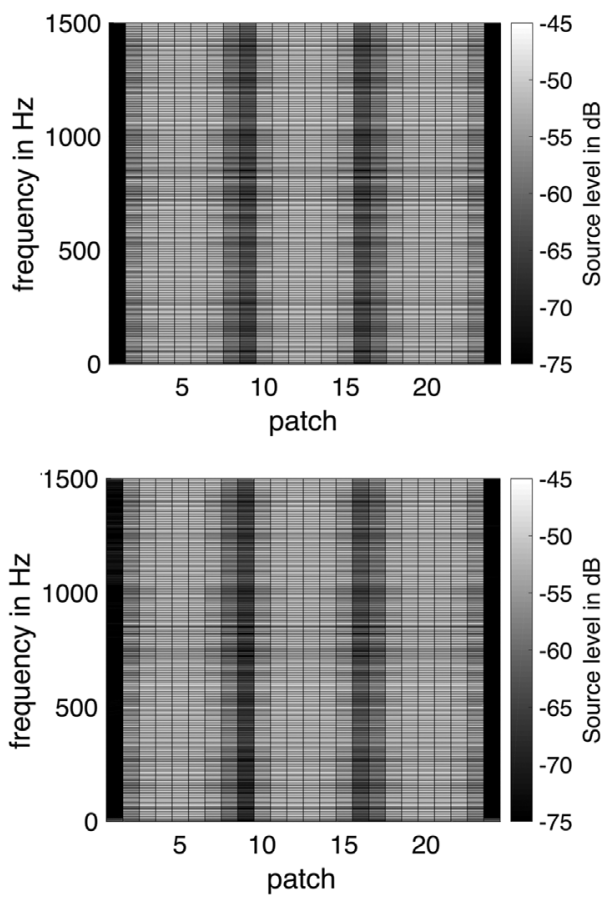

Figure 17. Comparison of the original source distribution along the 24 patches (upper) and the reconstructed source distribution (lower) as function of frequency for $N_{p}=3$. Reconstruction based on near-field receiving positions.

is given by modes on the structure. Having knowledge about the modes, one could directly identify the contribution of each mode to the vibration of the structure and to the radiated sound. Instead of structural modes of course any pattern can be used. In the case here the pattern given by (14) is utilised. For this $N_{p}=1$ to $N_{p}=10$ are considered. The impulse response functions for each of these patterns are calculated as,

$$
h_{N_{p}, r}=\sum_{m=0}^{24} v_{m}\left(N_{p}\right) \mathbf{h}_{m r}
$$

where $v_{m}$ is the prescribed velocity for a modal pattern according to (14) and $\mathbf{h}_{m, r}$ the impulse reaponse function between patch $m$ and receiving position $r$.

The reconstruction of a prescribed vibration pattern is based on two different sets of 36 receiving positions (one set in the near-field $10 \mathrm{~cm}$ above the plate and one set in the far-field on the half sphere). In the following the prescribed vibration consists of a combination of pattern according to (14) and the velocity at each patch can be written as,
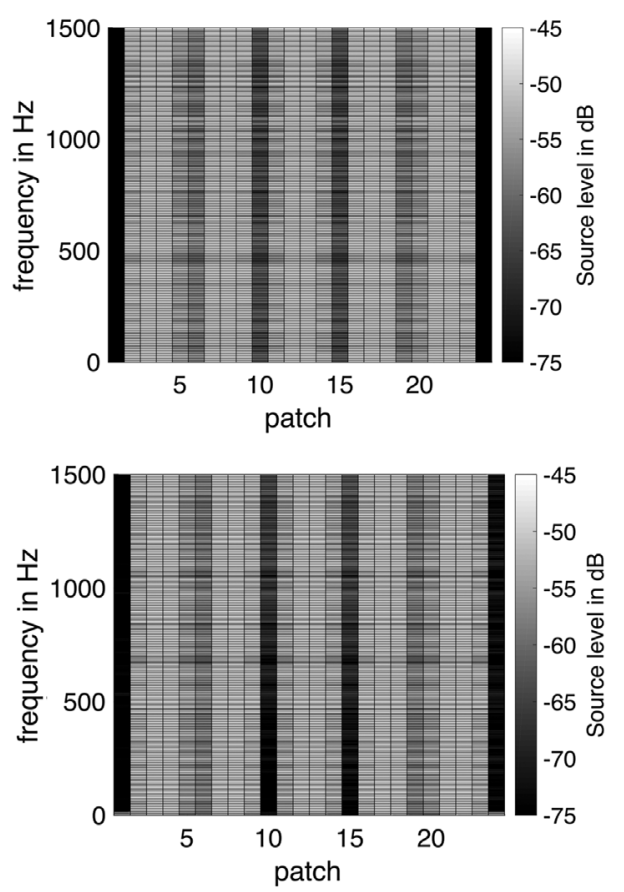

Figure 18. Comparison of the original source distribution along the 24 patches (upper) and the reconstructed source distribution (lower) as function of frequency for $N_{p}=5$. Reconstruction based on near-field receiving positions.

$$
v_{m}=\sum_{N_{p}=1}^{N_{p, \max }} A\left(N_{p}\right) \sin \left(\frac{N_{p} \pi m}{23}\right)
$$

where $A\left(N_{p}\right)$ is the amplitude for each pattern.

The reconstruction by means of far-field positions shows the same phenomena as in the previous section. Below the critical frequency given by the wavelength of the pattern $N_{p}$ the velocity distribution cannot be reconstructed correctly as only the part of the source distribution can be observed which is responsible for the radiated sound. Consequently, a possible source distribution is reconstructed which explains the radiated sound at the far-field receiving positions. Below $f_{c}$ the area in the middle of the radiating strip is contributing much less than the borders. However, due to the choice of the assumed base functions for the vibration pattern (i.e. sinusoidal functions), the patches at both ends always show zero velocity after reconstruction as shown in Figure 19. This also might indicate that the choice of the base of vibration patterns is not well adapted to the radiation problem although it certainly is the most appropriate to explain the vibrations on the plate.

In Figure 19 the amplitudes $A\left(N_{p}\right)$ are set to zero for all $N_{p}$ besides for $N_{p}=5$ which has unit amplitude. This is identical with the prescribed vibration patterns used in Figure 15. However, the results differ from the results shown there. This demonstrates the importance to use for the radiation relevant base functions and does not depend on the procedure of reconstructing the patterns. In the case considered here the sinusoidal function are not adequate. 

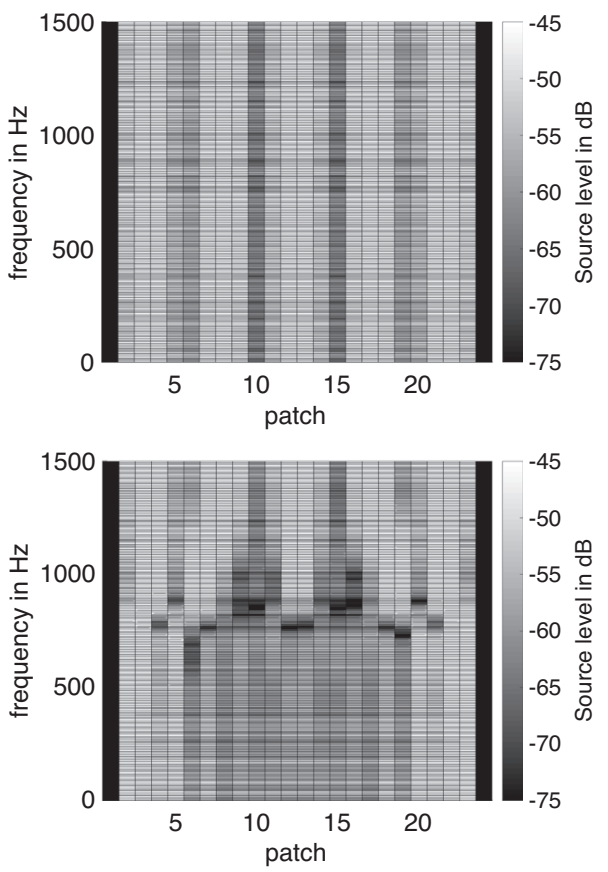

Figure 19. Comparison of the original source distribution along the 24 patches (upper) given by (16) and the reconstructed source distribution (lower) as function of frequency for $N_{p}=5$. Reconstruction based on far-field receiving positions.

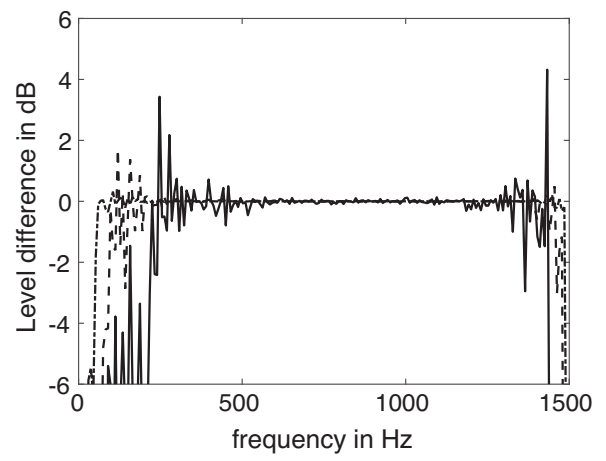

Figure 20. Level difference between original source strength and reconstructed source strength for pattern $N_{\mathrm{p}}=3$ for three different amplitudes: .- $10^{-4},--10^{-5},-10^{-6}$ and the amplitudes for the pattern $N_{p}=1,2,4,5$ set to one.

Similar problems might be observed when using modal patterns as base functions. The approach in 2.2.1 might be in this context clearly superior as it does not assume any pattern in advance.

The situation is again different when using near-field receiving positions for the reconstruction process. Results show that in the absence of measurement noise at the receiving positions the amplitudes $A\left(N_{p}\right)$ of the underlying patterns can be determined perfectly. This is true if the time signals for all patterns are correlated or uncorrelated and if their amplitudes are in the same order. Even if one single pattern is very small in amplitude in comparison to all other prescribed amplitudes the reconstruction works well over a wide frequency range as shown in Figure 20.

Figure 20 shows the level difference between original source strength and reconstructed source strength for the pattern $N_{p}=3$. The amplitude $A(3)$ is varied between $10^{-4}$ and $10^{-6}$ while the amplitudes for the pattern $N_{p}=1,2,4,5$ are set to one.

In these calculations all patterns have the same underlying time signal, i.e. all patterns are correlated but different in amplitude. In the presence of measurement noise the potential to reconstruct such weak source signals will strongly depend on the SNR. For decreasing SNR the possibility to reconstruct low amplitudes gets successively lost as expected. Similar results are obtained when assuming uncorrelated signals for all individual patterns.

\section{Application of the LMS algorithm to reconstruct a complex vibration pattern}

As final demonstration of the functioning of the LMS algorithm in the context of source identification the whole plate has a prescribed velocity pattern. This pattern is assumed to consist of sinusoidal functions similar to Equation (16) as,

$$
v_{x, y}=\sum_{n=1}^{4} \sum_{l=1}^{3} A(n, l) \sin \left(\frac{n \pi x}{L_{x}}\right) \sin \left(\frac{l \pi y}{L_{y}}\right),
$$

where $x$ and $y$ are the coordinates of the patch centre and $n$ and $l$ the number of half wavelengths fitting to the length and width respectively.

As amplitudes $A(n, l)$ the values were chosen randomly with the same order of magnitude (see Table 2 below).

Using near-field receiving positions allows to reconstruct the pattern on the plate rather well. However, to ensure a good agreement at low frequencies a high number of receiving positions is needed (see Fig. 21).

The differences mainly occur in nodal lines where the amplitudes are small which is typical for the LMS algorithm as it is controlled by the absolute error. However, looking at the averaged squared sound pressure level on the sphere which is proportional to the radiated sound power, the agreement between original values and the values obtained from the reconstructed source distribution are very close even if the reconstruction is based only on 12 receiving positions (see Fig. 22).

Using the far-field receiving positions for the reconstruction the results are very similar to the results in Section 2.2.

Figure 23 shows the results for the reconstruction based on 128 receiving positions for different frequencies. At low frequencies the pattern differs strongly from the original pattern shown in Figure 21. With increasing frequencies the differences decrease and at high frequencies the agreement is very good. However, looking at the averaged squared pressure on the sphere, the agreement is perfect over the whole frequency range. One can again conclude that the problem suffers from the fact that the same far-field pressure fields can be created by different source compositions. 
Table 2. Amplitudes $A(n, l)$ for the different patterns applied in Equation (17).

\begin{tabular}{lcccc}
\hline$l / n$ & 1 & 2 & 3 & 4 \\
\hline 1 & 1 & 0.2 & -0.5 & 1.2 \\
2 & -2 & -0.1 & 1.3 & 2 \\
3 & 1.5 & -1.1 & -0.7 & 0.65 \\
\hline
\end{tabular}
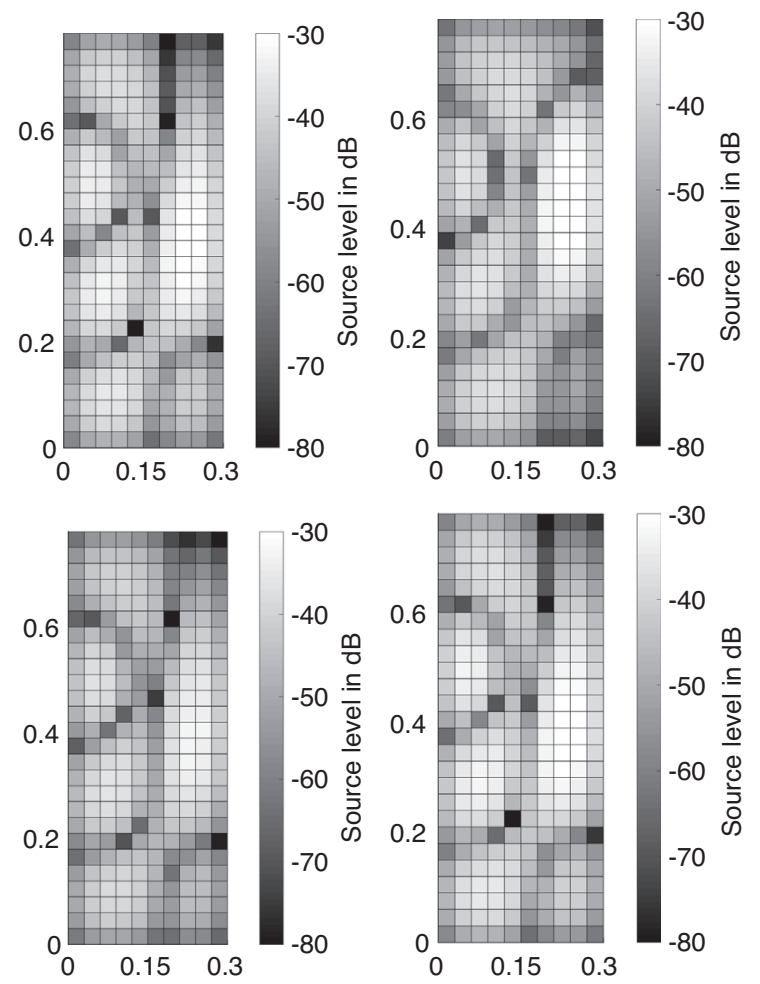

Figure 21. Comparison of the original pattern at $100 \mathrm{~Hz}$ (left upper corner) with the reconstructed pattern for 12 receiving position (right upper corner), 48 receiving position (left lower corner) and 64 receiving positions (right lower corner).

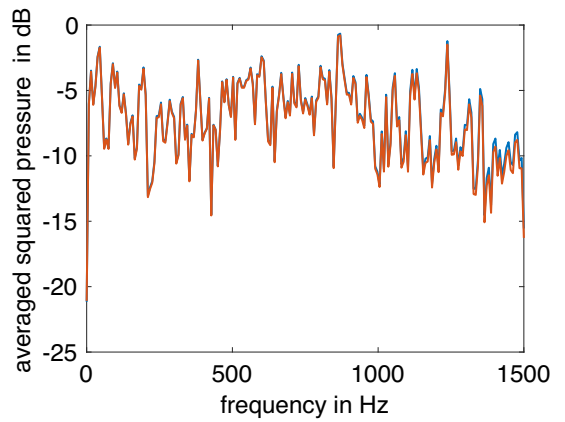

Figure 22. Averaged squared pressure on the half sphere for the original vibration pattern (solid gray line) and the reconstructed vibration pattern (dashed black line).

The problem is not the applied reconstruction method, but the underlying physics which makes it difficult to observe the required near-field information when the far-field is dominating.
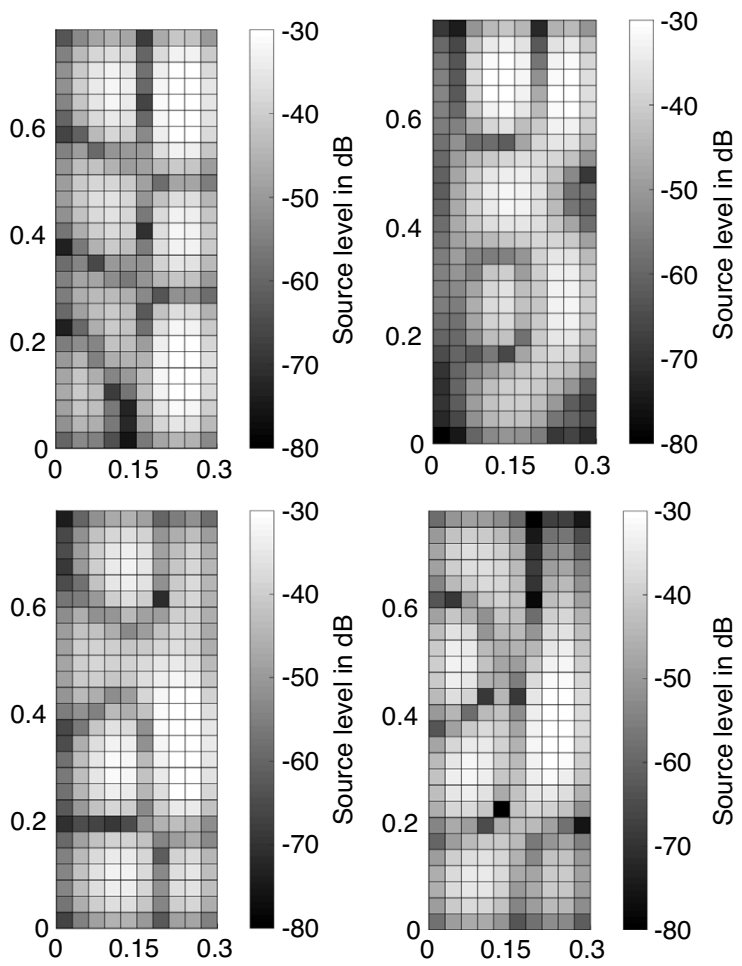

Figure 23. Results of the reconstructed vibration pattern at $100 \mathrm{~Hz}$ (left upper corner), $250 \mathrm{~Hz}$ (right upper corner), $500 \mathrm{~Hz}$ (left lower corner) and $1000 \mathrm{~Hz}$ (right lower corner).

\section{Conclusions}

A time domain approach for identifying the source distribution on a plate based on the LMS algorithm has been used. The approach has been demonstrated for SISO, SIMO and MIMO systems. A numerical experiment has been created as test case. FRFs have been calculated by means of a BE model describing the pressure at receiving positions normalised by the volume flow at discrete patches on the plate. Two sets of receiving positions were used, one in the far-field in the form of a half sphere around the centre of the plate and one in the near-field close to the surface of the plate. From the FRFs the IRFs were calculated as input to the LMS algorithm.

For the case of a single monopole represented by one of the patches on the plate the LMS algorithm demonstrated to be very robust. As for frequency domain approaches an overdetermined system (SIMO) can be formulated to suppress efficiently measurement noise as long as the noise is not correlated at the different receiving positions. A misspositioning of the source (i.e. the assumption of the wrong source position) showed to result in correct source signals as long as relevant receiving positions are utilised for the identification. This can be achieved by just choosing enough receiving positions. In the case considered here for a misspositioning of $15 \mathrm{~cm}$ the resulting error was still smaller than $1 \mathrm{~dB}$. Two different sets of receiving positions were utilised for the reconstruction of the vibration pattern on the plate. 
The first set consisted of receiving positions in the nearfield of the source on the plate. The examples in this paper showed that in this case accurate reconstruction is achievable as long as sufficient many observation positions are utilised. It was shown that the so reconstructed source distribution creates the same far-field sound field as the original distribution.

The second set consisted of receiving positions in the far-field of the sources on the plate. In this case the reconstruction leads to source distributions which at lower frequencies clearly differ from the original source distributions. This is due to the fact that in the far-field only the part of the source pattern can be observed that is responsible for the radiated sound. At the end it is not a failure of the approach, but a consequence of the underlying physics which determines the limits of the reconstruction as the required near-field information is hardly to be extracted when the far-field is dominating.

Referring back to the list of typical sources of errors as mentioned in the introduction of this paper one can conclude the following for the topics considered in this paper:

- Insufficient signal to noise ratio which might lead to erroneous observation of field quantities (e.g. measured vibrations or sound pressures) or frequency response functions (FRFs) can be improved by an overdetermined system. The LMS algorithm is extremely robust with respect to measurement noise due to the fact that it is controlled by the strongest signals which has the highest SNR. In addition as long as the measurement noise is not correlated to the signals to be reconstructed, even erroneous gradients in the updating process are random and averaged out over the iteration process.

- Non-accurate FRFs due to e.g. small misplacement of transducers or the inherent difficulties to measure certain degrees of freedom (DoF) such as rotational degrees of freedom. The LMS algorithm showed to be capable even for this case to find correct source signals as long as sufficiently relevant receiving position are used.

- Neglecting the presence of correlated multiple sources. Multiple correlated sources might lead to crosstalking at interfaces. Neglecting some of the source will give erroneous results for those sources taken into account. This has turned out not to be a problem in the case considered here. Assuming several correlated sources did not create any problems for the identification process. Even correlated sources with substantially low amplitudes could be detected as long as the background noise is not too high and therefore masking the contribution of low amplitude sources.

In practical applications it will be required either to calculate or to measure a complete set of impulse response functions between the expected sources patches and or vibration pattern (e.g. modes) and observation positions. Calculation using e.g. the boundary element method will be very efficient but often demand simple situations (e.g. anechoic conditions) to keep the computational effort acceptable. Measurement in contrary can include the complexity of the sound field. It can be expected that the complexity of a sound field in a room will improve the efficiency of the method as it will increase the difference between individual impulse response functions as long as the reverberation will not become too high.

In some way the presented approach is similarto NAH or IBEM as it uses pre-knowledge about the sound field to reconstruct the source distribution. The big advantage of the approach, however, is that does not demand certain geometries and no inversion is required and therefore typical problems of inverse methods are avoided. In addition the time domain formulation is perfectly for transient signals and time varying source distributions.

Finally, there is the limitation by lack of information due to the physics of the problem might not allow for identifying sources or transfer paths accurately. In the case considered here, it has been demonstrated that the observation of the sound field in the far-field is not sufficient for reconstructing the source distribution if the sources mainly create a near-field. In this case it is essential to place observation positions in the near-field to be able to "see" the correct source distribution.

\section{References}

1.W.J. Verheij: Multi-path sound transfer from resiliently mounted shipboard machinery: Experimental methods for analyzing and improving noise control. Ph.D. Thesis at Delft University of Technology, The Netherlands, 1982.

2. M. Van der Seijs, D. de Klerk, D.J. Rixen: General framework for transfer path analysis: History, theory and classification of techniques. Mechanical Systems and Signal Processing 68-69 (2016) 217-244.

3. G. Williams, J.D. Maynard: Numerical evaluation of the Rayleigh integral for planar radiators using the FFT. Journal of the Acoustical Society of America 72 (1982) 20202030.

4. J.D. Maynard, E.G. Williams, Y. Lee: Nearfield acoustic holography: I. Theory of generalized holography and the development of NAH. Journal of the Acoustical Society of America 78 (1985) 1395-1413.

5. G.-T. Kim, B.-T. Lee: 3-D sound source reconstruction and field reproduction using the Helmholtz integral equation. Journal of Sound and Vibration 136 (1990) 245-261.

6. Y.-C. Chao: An implicit least-squares method for the inverse problem of acoustic radiation. Journal of the Acoustical Society of America 81 (1987) 1288-1292.

7. Z. Wang, S.F. Wu: Helmholtz equation-least-squares method for reconstructing the acoustic pressure field. Journal of the Acoustical Society of America 102 (1997) 2020-2032.

8. A.S. Elliott, A.T. Moorhouse: Characterisation of structure borne sound sources from measurement in-situ. Journal of the Acoustical Society of America 123, 5 (2008) 3176.

9. J.M. Mondot, B.A.T. Petersson: Characterization of structureborne sound sources: the source descriptor and the coupling function. Journal of Sound and Vibration 114, 3 (1987) 507-518.

10.D. De Klerk, D.J. Rixen: Component transfer path analysis method with compensation for test bench dynamics. Mechanical Systems and Signal Processing 24, 6 (2010) 1693-1710. 
11. B.J. Dobson, E. Rider: A review of the indirect calculation of excitation forces from measured structural response data. Proceedings of the Institution of Mechanical Engineers, Part C: Journal of Mechanical Engineering Science 204-2 (1990) 69-75.

12. A.N. Thite, D.J. Thompson: The quantification of structureborne transmission paths by inverse methods. Part 2: Use of regularization techniques. Journal of Sound and Vibration 264, 2 (2003) 433-451.

13. A.N. Thite, D.J. Thompson: The quantification of structureborne transmission paths by inverse methods. Part 1: Improved singular value rejection methods. Journal of Sound and Vibration 264, 2 (2003) 411-431.

14. W. Kropp, K. Larsson: Force estimation in the time domain by applying an LMS-algorithm, in Proceedings NOVEM (2005).

15. M. Sturm, A.T. Moorhouse, W. Kropp, T. Alber: Robust calculation of simultaneous multi-channel blocked force signatures from measurements made in-situ using an adaptive algorithm in time domain, in Bangkok, Thailand, 2013.
16. N. Amiryarahmadi, W. Kropp, K. Larsson: Application of the LMS algorithm to measure low-frequency transient forces from human walking. Acta Acustica United with Acustica 202, 1 (2016) 23-34.

17. H. Brick: Application of the Boundary Element Method to combustion noise and half-space problems. Ph.D. Thesis, Chalmers University of Technology, Göteborg, Sweden, 2009.

18. B. Widrow, M. Hoff: Adaptive switching circuits. Proceedings IRE Wescon Convention Record, Part 4 Session 16 (1960) 96-104.

19. B. Widrow, J.M. Cool, M.G. Larimore, C.R. Johnson: Stationary and non-stationary learning characteristics of the LMS adaptive filter. Proceedings of the IEEE 64, 8 (1976) 1151-1162.

20. R.J. Allemang: The modal assurance criterion - twenty years of use and abuse. Sound and Vibration 37, 1, (2003) 14-23.

21. F. Fahy: Sound and structural vibration. Academic Press, 1985 , p. 86.

Cite this article as: Kropp W. Larsson K. Lobato T. \& Sottek R. 2021. Application of the LMS algorithm to identify the surface velocity responsible for the radiated sound pressure. Acta Acustica, 5, 32. 